\title{
Odontopediatras e técnicas aversivas no controle do comportamento infantil
}

Pediatric dentists and aversive techniques for child behavior control

\author{
Talita Barbosa Minhoto* \\ Matheus de França Perazzo ${ }^{* *}$ \\ Érick Tássio Barbosa Neves ${ }^{* * *}$ \\ Ana Flávia Granville-Garcia*** \\ Bianca Oliveira Tôrres ${ }^{* * * *}$ \\ Jainara Maria Soares Ferreira ${ }^{* * * * *}$
}

\section{Resumo}

Objetivo: avaliar o perfil dos odontopediatras e o uso das técnicas aversivas para o controle do comportamento no cotidiano clínico. Sujeitos e método: foi realizado um estudo transversal, censitário, realizado com 33 odontopediatras atuantes na cidade de João Pessoa, regularmente inscritos no Conselho Regional de Odontologia da Paraíba. Os dados foram coletados por meio de questionário, objetivando avaliar: dados sociodemográficos; seleção e indicação das técnicas aversivas; ansiedade do odontopediatra e o consentimento e aceitação das técnicas aversivas pelas crianças/responsáveis. A análise dos dados foi realizada por estatística descritiva. Resultados: os odontopediatras foram representados principalmente pelo sexo feminino (93,9\%), graduados há mais de 10 anos $(84,8 \%)$ e com especialização $(45,5 \%)$. Entre os profissionais, $84,8 \%$ utilizavam as técnicas aversivas, destes, $75,0 \%$ utilizavam as técnicas em pelo menos dois a cada 10 pacientes. No entanto, apenas $66,6 \%$ consideraram a abordagem do assunto suficiente durante a pós/graduação. A técnica aversiva mais escolhida pelos profissionais foi a contenção de braços, pernas e cabeça sem dispositivos específicos (53,6\%), a principal indicação foi para crianças birrentas e agressivas $(39,3 \%)$ e os profissionais relataram baixa ansiedade para execução das técnicas $(46,4 \%)$. A maioria dos odontopediatras $(60,7 \%)$ não solicitava a assinatura do termo de consentimento. Além disso, 92,9\% afirmaram que a estabilização protetora não era rejeitada pelos pais, como também, 92,9\% não consideraram que a estabilização protetora prejudicasse a aceitação do tratamento odontológico posterior da família do paciente. Conclusão: foi comum os odontopediatras utilizarem as técnicas aversivas para o controle comportamental, porém poucos solicitavam a autorização pelo termo de consentimento.

Palavras-chave: Odontopediatria. Comportamento infantil. Terapia aversiva. Controle Comportamental. Odontólogo.

\section{Introdução}

O ambiente odontológico pode trazer ansiedade às crianças e um consequente comportamento negativo, o que dificulta a execução dos procedimentos clínicos ${ }^{1,2}$. Diante disto, técnicas de controle do comportamento infantil podem auxiliar no atendimento, no sentido de desenvolver na criança um comportamento mais apropriado enquanto recebe o tratamento, ajudando a criança a aprender, a entender e a cooperar na cadeira odontológica ${ }^{3,4}$. A técnica não aversiva é a primeira escolha de intervenção frente ao paciente não cooperativo, a exemplo da comunicação não verbal, reforço positivo, distração ${ }^{1,5,6}$.

No entanto, em determinadas situações, as técnicas não aversivas não são suficientes e recorrer a uma medida farmacológica pode ser pouco viável ou inacessível ${ }^{7}$.

As técnicas de controle comportamental aversivas são indicadas em situações em que há necessidade da estabilização protetora como medida preventiva da integridade dos envolvidos ${ }^{8,9}$. A técnica clássica de controle aversivo do paciente infantil é a estabilização protetora, que pode ser parcial ou total ${ }^{9,10}$. A utilização apropriada da técnica é capaz de melhorar o comportamento do paciente nas próximas visitas ao consultório odontológico, fazendo com que o uso de técnicas menos invasivas seja introduzido sucessivamente ${ }^{11}$.

Cabe ao cirurgião-dentista estabelecer critérios adequados para selecionar a técnica aversiva como uma forma de intervenção ${ }^{8}$. A escolha da técnica mais apropriada é definida pelo contex- 
to de aplicação, necessidade do paciente e habilidades do praticante ${ }^{10,12,13}$. A escolha também depende de fatores como a cultura local, fatores educacionais, preparo para executar as técnicas e questões legais e éticas ${ }^{5,13,14}$.

Estudos relacionados às técnicas aversivas, frequentemente, abordam sobre os tipos de técnicas e a receptibilidade das crianças e responsáveis ${ }^{6,9,15,16}$. Ao serem pesquisados os unitermos "child-management techniques AND pediatric dentists", "aversive therapy AND pediatric dentistry", "terapia aversiva AND odontólogos" nas bases da SciELO e PubMed, foram encontrados poucos estudos que buscaram avaliar a visão prática e a rotina dos odontopediatras quanto às técnicas aversivas ${ }^{1,17}$. Portanto, o objetivo do presente estudo foi avaliar o perfil dos odontopediatras e os tipos e repercussões das técnicas de controle comportamental aversivas empregadas no cotidiano clínico.

\section{Sujeitos e método}

Foi realizado um estudo transversal com 44 odontopediatras regulares no Conselho Regional de Odontologia da Paraíba (CRO-PB), em João Pessoa-PB. João Pessoa é uma cidade industrializada do nordeste brasileiro, com Índice de Desenvolvimento Humano (IDH) de $0,763^{18}$. A coleta foi censitária porque o número de odontopediatras regulamentados no CRO-PB em João Pessoas era inferior a 250 indivíduos, portanto, é sugerido que toda a população seja avaliada ${ }^{19}$.

A coleta dos dados foi realizada na forma de entrevista com os odontopediatras no ambiente de trabalho, em horário agendado. O questionário aplicado abordou questões relacionadas à caracterização sociodemográfica da população; frequência e o tipo de técnica aversiva utilizada pelos odontopediatras; perfil das crianças em que era utilizada a estabilização protetora; nível de ansiedade do profissional para realizar as técnicas aversivas; solicitação de autorização verbal e/ou do termo de consentimento para realizar a estabilização protetora; repercussões do uso de técnicas aversivas sobre a receptibilidade dos tratamentos odontológicos posteriores e a aceitação dos pais diante da indicação das técnicas aversivas.
No estudo piloto, a avaliação do entendimento do questionário por parte dos cirurgiões-dentistas foi realizada por meio do método de "validação de face" com $10 \%$ da população ${ }^{20}$. Para isso, o pesquisador pediu que os entrevistados explicassem com as próprias palavras o que entenderam para cada item. Nenhum odontopediatra apresentou dúvida ou dificuldade para responder aos itens do questionário. A confiabilidade teste-reteste foi determinada após um intervalo de sete dias entre a aplicação dos questionários com os mesmos indivíduos. A concordância entre os testes foi de 92,8\%. A administração dos questionários levou aproximadamente 5 minutos. Os participantes do piloto foram incluídos no estudo principal.

Este estudo recebeu a aprovação pela $80^{\underline{a}}$ reunião ordinária do Comitê de Ética em Pesquisa do Unipê, com base na Resolução 466/2012 do Conselho Nacional de Saúde (CNS), sob o protocolo $\mathrm{n}^{\circ}$ 499/2015 e certificado de apresentação para Apreciação Ética: 42617315.1.0000.5176. Foi garantida a autonomia à escolha de participação a todos os indivíduos. Os odontopediatras leram e assinaram o Termo de Consentimento Livre e Esclarecido.

Após a coleta dos dados, categorização das variáveis e dados coletados, foi realizada a transferência desses para um banco de dados informatizado no pacote estatístico SPSS (Statistical Package for the Social Sciences) versão 20.0. Os dados foram avaliados por estatística descritiva.

\section{Resultados}

Um total de 33 odontopediatras participaram do estudo. A perda de $25,0 \%$ da população foi devido à recusa na participação do estudo (7) e por motivos de aposentadoria (4). As características sociodemográficas e o contato com as técnicas aversivas na prática e formação profissional estão na Tabela 1. A população de odontopediatras foi composta principalmente pelo sexo feminino $(93,9 \%)$, com idade entre 30 e 50 anos $(54,5 \%)$ e formação acadêmica concluída há mais de 10 anos $(84,8 \%)$; especialistas em Odontopediatria $(45,5 \%)$; com suficiente abordagem das técnicas aversivas durante a graduação e/ou pós-graduação $(66,6 \%)$ e que utilizavam as técnicas no cotidiano clínico $(84,8 \%)$. 
Tabela 1 -Características sociodemográficas e familiaridade dos odontopediatras com as técnicas aversivas para o controle do comportamento.

\begin{tabular}{|c|c|c|}
\hline Variáveis & $\mathrm{N}$ & $\%$ \\
\hline \multicolumn{3}{|l|}{ Idade } \\
\hline$\leq 30$ anos & 2 & 6,1 \\
\hline$>30$ anos $\mathrm{e} \leq 50$ anos & 18 & 54,5 \\
\hline$>50$ anos & 13 & 39,4 \\
\hline \multicolumn{3}{|l|}{ Sexo } \\
\hline Masculino & 2 & 6,1 \\
\hline Feminino & 31 & 93,9 \\
\hline \multicolumn{3}{|l|}{ Tempo de formado } \\
\hline$\leq 10$ anos & 5 & 15,2 \\
\hline$>10$ anos & 28 & 84,8 \\
\hline \multicolumn{3}{|l|}{ Título acadêmico } \\
\hline Especialista & 15 & 45,5 \\
\hline Mestre & 5 & 15,2 \\
\hline Doutor & 13 & 39,4 \\
\hline \multicolumn{3}{|c|}{ Abordagem das técnicas aversivas na pós/graduação } \\
\hline Suficiente & 22 & 66,6 \\
\hline Insuficiente & 10 & 30,3 \\
\hline Não houve & 1 & 3,1 \\
\hline \multicolumn{3}{|l|}{ Utiliza técnicas aversivas } \\
\hline Sim & 28 & 84,8 \\
\hline Não & 5 & 15,2 \\
\hline
\end{tabular}

Na Tabela 2, são encontradas as respostas dos odontopediatras para as questões relacionadas às técnicas aversivas de controle de comportamento. As respostas foram divididas em três grandes domínios, de acordo com o tema abordado: seleção e indicação das técnicas aversivas; ansiedade do odontopediatra e consentimento das técnicas aversivas; aceitação das técnicas aversivas. Para o domínio a seleção e a indicação das técnicas aversivas, foi observado que a técnica aversiva mais utilizada entre os odontopediatras foi a contenção de braços, pernas e cabeça sem dispositivos específicos $(53,6 \%)$. Além disso, quase metade dos profissionais $(46,4 \%)$ utilizava alguma técnica aversiva em 1 a cada 10 pacientes, principalmente nas crianças com comportamento birrento e agressivo (39,3\%). No segundo domínio, foi encontrado a baixa ansiedade do odontopediatra diante de procedimentos que exigiam técnicas aversivas $(96,3 \%)$. Para tais procedimentos, a grande maioria dos profissionais pediam autorização verbal $(96,3 \%)$, no entanto, apenas $39,3 \%$ solicitavam a assinatura do termo de consentimento. Quanto ao domínio sobre aceitação das técnicas aversivas, os odontopediatras afirmaram que os pais não rejeitavam o uso de técnicas de estabilização protetora $(92,9 \%)$. Além disso, a estabilização protetora não atrapalhava a aceitação do tratamento odontológico posterior da família do paciente $(92,9 \%)$.
Tabela 2 - Técnicas aversivas para o controle do comportamento no cotidiano clínico dos odontopediatras.

\begin{tabular}{|c|c|c|}
\hline \multicolumn{3}{|c|}{ Seleção e indicação das técnicas aversivas } \\
\hline Questões & $\mathrm{N}$ & $\%$ \\
\hline \multicolumn{3}{|l|}{$\begin{array}{l}\text { Das técnicas descritas a seguir, qual você } \\
\text { mais usa? }\end{array}$} \\
\hline $\begin{array}{l}\text { Contenção de braços, pernas e cabeça } \\
\text { sem dispositivos específicos }\end{array}$ & 15 & 53,6 \\
\hline $\begin{array}{l}\text { Abertura de boca com dispositivos } \\
\text { específicos }\end{array}$ & 10 & 35,7 \\
\hline Mão sobre a boca & 3 & 10,7 \\
\hline \multicolumn{3}{|l|}{$\begin{array}{l}\text { A cada } 10 \text { pacientes, em quantos você } \\
\text { usa a estabilização protetora? }\end{array}$} \\
\hline 1 & 13 & 46,4 \\
\hline 2 & 8 & 28,6 \\
\hline 3 & 3 & 10,7 \\
\hline 4 & 2 & 7,1 \\
\hline 5 & 1 & 3,6 \\
\hline 10 & 1 & 3,6 \\
\hline \multicolumn{3}{|l|}{$\begin{array}{l}\text { Qual(is) o(s) perfil(is) da(s) da criança que } \\
\text { você usa a estabilização protetora? }\end{array}$} \\
\hline Birrenta & 8 & 28,6 \\
\hline Agressiva & 5 & 17,9 \\
\hline Birrenta e agressiva & 11 & 39,3 \\
\hline Medrosa, ansiosa, birrenta e agressiva & 1 & 3,6 \\
\hline Medrosa e birrenta & 1 & 3,6 \\
\hline
\end{tabular}

\begin{tabular}{l|c|c}
\hline \multicolumn{3}{c|}{$\begin{array}{l}\text { Ansiedade do odontopediatra e consentimento } \\
\text { das técnicas aversivas }\end{array}$} \\
\hline \multicolumn{4}{c|}{ Questões } & $\mathrm{n}$ & $\%$
\end{tabular}

Como você classificaria a sua ansiedade em realizar um procedimento com técnicas aversivas para controle de comportamento infantil?

Muito alta

Alta

Intermediária

Baixa

Muito baixa

Você solicita a autorização verbal aos pais das crianças quando usa a estabilização protetora?

Sim

Não

Você solicita a assinatura do termo de consentimento aos pais das crianças quando usa a estabilização protetora?

Sim

$$
\text { Não }
$$

\begin{tabular}{|c|c|c|}
\hline \multicolumn{3}{|c|}{ Aceitação das técnicas aversivas } \\
\hline Questões & $\mathrm{n}$ & $\%$ \\
\hline $\begin{array}{l}\text { Os pais, frequentemente, rejeitam o uso } \\
\text { da estabilização protetora para os filhos? }\end{array}$ & & \\
\hline Sim & 2 & 7,1 \\
\hline Não & 26 & 92,9 \\
\hline $\begin{array}{l}\text { Você considera que a estabilização pro- } \\
\text { tetora para controle do comportamento } \\
\text { na Odontopediatria repercute negativa- } \\
\text { mente na aceitação do tratamento odon- } \\
\text { tológico posterior da família do paciente? }\end{array}$ & & \\
\hline Sim & 2 & 7,1 \\
\hline Não & 26 & 92,9 \\
\hline
\end{tabular}




\section{Discussão}

O presente estudo avaliou o uso de técnicas aversivas para o controle do comportamento por odontopediatras. A população de odontopediatras foi representada predominantemente pelo sexo feminino $(93,9 \%)$, característica comum à especialidade ${ }^{21}$. Ademais, profissionais mostraram ser experientes na área, pois a maioria apresentou mais de 10 anos de formado $(84,8 \%)$ e com título de especialista em Odontopediatria $(45,5 \%)$. No entanto, o reduzido número de odontopediatras com 10 anos de formado ou menos $(15,2 \%)$, sugere uma queda pela procura da especialidade nos últimos anos na região. Logo, pode representar um risco à saúde bucal e à qualidade de vida das crianças e das famílias ${ }^{22,23}$.

Os cirurgiões-dentistas recém-formados costumam utilizar técnicas não aversivas ${ }^{3,9}$. A preferência por técnicas menos invasivas está relacionada ao crescente conhecimento dos odontopediatras sobre a psicologia infantil ${ }^{12,24-26}$. No presente estudo, poucos participantes eram recém-formados, o que pode justificar o fato da grande maioria da população utilizar técnicas aversivas no cotidiano clínico $(84,8 \%)$. Além disso, a frequência da aplicação da técnica aversiva era comum entre os participantes, já que 75,0\% dos cirurgiões-dentistas utilizavam as técnicas em pelo menos dois a cada 10 pacientes. No entanto, $33,4 \%$ dos odontopediátras consideraram a abordagem das técnicas aversivas insuficiente/ inexistente durante a graduação ou pós-graduação, o que pode repercutir na aplicação inadequada da técnica. Diante disso, a educação continuada sobre o gerenciamento do comportamento infantil pode representar uma estratégia eficiente, visto que os conhecimentos éticos e práticos da técnica influenciam o êxito do atendimento clínico na Odontopediatria 8,27 .

A contenção de braços, pernas e cabeça sem dispositivos específicos foi a técnica aversiva mais utilizada entre os odontopediatras $(53,6 \%)$. A contenção é indicada para pacientes que necessitam de tratamento imediato, porém que não cooperam por imaturidade emocional ou pela condição física e mental ${ }^{8,10,25}$. Neste estudo, 39,3\% dos odontopediátras consideravam o comportamento birrento e agressivo como condições em que são indicados a estabilização protetora. No entanto, a estabilização do paciente é um assunto controverso. $\mathrm{O}$ uso da contenção pode repercutir em prejuízos físicos e psicológicos, perda da dignidade e até na violação dos direitos do paciente ${ }^{9,24}$. Mesmo com os riscos, a maioria dos odontopediátras $(57,1 \%)$ relatou baixa/ muito baixa ansiedade para realizar um procedimento com técnicas aversivas. Assim, a baixa ansiedade sugere que os participantes do estudo possuem um considerável domínio das estratégias para o controle comportamental na clínica ${ }^{26}$.
É dever do odontopediatra pedir o consentimento dos responsáveis pela criança antes de executar qualquer técnica aversiva, exceto em casos de urgência ${ }^{14}$. A autorização precisa ser documentada e anexada ao prontuário do paciente ${ }^{8,10}$. Porém, no presente estudo, apenas $39,3 \%$ dos odontopediátras solicitavam a assinatura do termo de consentimento, já os demais, não pediam permissão ou apenas solicitavam a autorização verbal. Não solicitar a autorização infringe o Código de Ética Odontológica e desampara legalmente os odontopediatras em casos de intercorrências ${ }^{14}$. Machado et al. ${ }^{27}$, encontraram resultados semelhantes, de forma que a autorização verbal correspondeu a $70,2 \%$, enquanto a assinatura do termo de consentimento representou 5,3\%.

Quanto à aceitação das técnicas aversivas, $92,9 \%$ dos odontopediatras relataram que o uso das manobras não era rejeitado pelos pais. No entanto, estudos mostram que pais preferem as técnicas menos invasivas, como o controle da voz e a técnica dizer-mostra-fazer ao invés da estabilização protetora $^{5,28}$. A diferença entre os resultados pode ser justificada pelo fato do presente estudo não ter objetivado avaliar diretamente a opinião do pai, mas, sim, a percepção dos odontopediátras. Caso a utilização técnica aversiva seja necessária, os pais tendem a uma maior aceitação quando previamente informados sobre as manobras de controle comportamental ${ }^{21}$. Esta conscientização pré-intervenção dos pais pode ser o motivo de ter sido encontrado a aceitação da família para tratamentos posteriores de $92,9 \%$.

O presente estudo apresenta limitações inerentes ao estudo transversal, de forma que as respostas dadas pelas mães podem estar sujeitas ao viés de informação. Além disto, possui apenas validade para a população do estudo.

Diante dos resultados encontrados, é recomendado cursos de atualização em Odontopediatria com foco nas técnicas não aversivas, enfatizando as questões éticas na seleção da manobra. A avaliação da visão do próprio odontopediátra sobre as técnicas de controle do comportamento permite identificar as fragilidades da prática clínica, portanto é recomendada a realização de novos estudos em outras regiões, com diferentes perfis de formação.

\section{Conclusão}

A população de odontopediátras incluída neste estudo foi predominantemente composta por profissionais do sexo feminino que possuíam conhecimento sobre as técnicas aversivas adquirido durante a sua formação. A técnica aversiva mais utilizada pelos cirurgiões-dentistas foi a contenção de braços, pernas e cabeça sem a utilização de dispositivos específicos para esta finalidade. Embora de grande importância, a aplicação com os pais de um termo de consentimento livre e esclarecido como pré-re- 
quisito legal para execução de técnicas aversivas nas crianças, foi menos utilizada em detrimento à autorização meramente verbal. Para a população deste estudo, o uso de técnicas aversivas parece representar, quando corretamente indicado, uma opção viável de controle do comportamento infantil, com pouca repercussão negativa na aceitação da criança com relação aos tratamentos posteriores e boa receptividade por parte dos responsáveis.

\section{Abstract}

Objective: to evaluate the profile of pediatric dentists and the use of aversive techniques for behavior control in the clinical routine. Subjects and method: it was a cross-sectional census study conducted with 33 pediatric dentists working in the city of João Pessoa, $P B$, Brazil, regularly enrolled in the Regional Council of Dentistry of the state of Paraíba. Data were collected through a questionnaire, aiming to evaluate sociodemographic data, selection and indication of aversive techniques, anxiety of the pediatric dentist, and consent and acceptance of aversive techniques by children/ caregivers. Data was analyzed by descriptive statistics. Results: pediatric dentists were represented mostly by women $(93.9 \%)$ graduated for over 10 years (84.8\%) and specialized in the field (45.5\%). Among the professionals, $84.8 \%$ used aversive techniques; from these, $75.0 \%$ used the techniques in at least two out of 10 patients. However, only $66.6 \%$ consider the topic to be sufficiently addressed during post/graduate courses. The aversive technique chosen by most professionals was the restraint of arms, legs, and head without specific devices (53.6\%), mainly used in obstinate and aggressive children (39.3\%) and performed with low anxiety by professionals (46.4\%). Most pediatric dentists (60.7\%) had not requested the signing of the consent form. In addition, $92.9 \%$ reported the parents did not reject protective stabilization techniques; also 92.9\% did not consider that protective stabilization would interfere with acceptance of subsequent dental treatment from the patient's family. Conclusions: it was common for pediatric dentists to use aversive techniques for behavioral control, but few of them requested authorization through a consent form.

Keywords: Pediatric Dentistry. Child Behavior. Aversive Therapy. Behavior Control. Dentist.

\section{Referências}

1. Peretz B, Glaicher H, Ram D. Child-management techniques. Are there differences in the way female and male pediatric dentists in Israel practice? Braz Dent J 2003; 14(2):82-6.

2. Bottan ER, Trentini L, Araújo SM. Ansiedade no tratamento odontológico: levantamento em estudantes do ensino fundamental do município de Pouso Redondo - SC. RFO 2007; 12(3):7-12.

3. Zhou Y, Humphris GM. Reassurance and distress behavior in preschool children undergoing dental preventive care procedures in a community setting: a multilevel observational study. Ann Behav Med 2014;48(1):100-11.
4. Seger, L. Psicologia e odontologia: uma visão integradora. 3.ed. São Paulo: Santos; 1998. 165p.

5. Al-Jobair AM, Al-Mutairi MA. Saudi dental students' perceptions of pediatric behavior guidance techniques. BMC Medical Education. 2015;15:120.

6. Navit S, Johri N, Khan SA, Singh RK, Chadha D, Navit P, et al. Effectiveness and Comparison of Various Audio Distraction Aids in Management of Anxious Dental Paediatric Patients. J Clin Diagn Res 2015;9(12):5-9.

7. Roberts JF, Curzon MEJ, Koch G, Martens LC. Review: Behaviour Management Techniques in Paediatric Dentistry. European Archives of Paediatric Dentistry 2010; 11(4):166-74.

8. Barbosa CSA, Toledo AO. Uso de técnicas aversivas de controle de comportamento em Odontopediatria. Rev. IberoAm. Odontopediatr. Odontol. Bebê 2003; 6(29):76-82.

9. Oliver K, Manton DJ. Contemporary behavior management techniques in clinical pediatric dentistry: out with the old and in with the new? J Dent Child (Chic) 2015;82(1):22-8.

10. American Academy of Pediatric Dentistry. Guideline on behavior guidance for the pediatric dental patient. Reference Manual. Pediatr Dent 2015;36:179-91.

11. Sharath A, Rekka P, Muthu MS, RathnaPrabhu V, Sivakumar N. Children's behavior pattern and behavior management techniques used in a structuredpostgraduate dental program. J Indian Soc Pedod Prevent Dent 2009;27(1):22-6.

12. Albuquerque CM, Gouvêia CVD, Moraes RCM, Barros RN, Couto CF. Principais técnicas de controle de comportamento em Odontopediatria. Arquivos em Odontologia 2010;45 (2):110-15

13. Juntgen LM, Sanders BJ, Walker LA, Jones JE, Weddell JA, Tomlin AM, et al. Factors influencing behavior guidance: a survey of practicing pediatric dentists. Pediatr Dent 2013;35(7):539-45.

14. Conselho Federal de Odontologia. Código de Ética Odontológica, 2012. Resolução CFO, v. 118, 2012.

15. Wilson S. Management of child patient behavior: quality of care, fear and anxiety, and the child patient. Pediatr Dent. 2013;35(2):170-4.

16. Patel M, McTigue DJ, Thikkurissy S, Fields HW. Parental Attitudes Toward Advanced Behavior Guidance Techniques Used in Pediatric Dentistry. Pediatr Dent 2016;38(1):30-6.

17. Crossley ML, Joshi G. An investigation of paediatric dentists' attitudes towards parental accompaniment and behavioural management techniques in the UK. BrDent $\mathrm{J}$ 2002;192(9):517-21.

18. Instituto Brasileiro de Geografia e Estatística. Brasília (DF): Instituto Brasileiro de Geografia e Estatística. [cited 2016 Feb 15] Available from: http://cidades.ibge.gov.br/ xtras/home.php.

19. Antunes JLF, Peres MA. O método epidemiológico de investigação e sua contribuição para a saúde bucal. In: Antunes JLF, Peres MA. Fundamentos de Odontologia: Epidemiologia da Saúde Bucal. Guanabara Koogan: Rio de Janeiro; 2006. p 2-18.

20. Frankfort-Nachimias C, Nachimias D. Research methods in the social sciences. 4. ed. London: Edward Arnold; 1992.

21. Farias IAP, Costa MRM, Oliveira AFB, Pereira AMBC; Massoni, ACLT. Professional profile analysis and college education of pediatric dentistry and group of general dentistry of João Pessoa, Paraiba, Brazil. Rev odontol UNESP 2010;39(1):27-31.

22. Gomes MC, Pinto-Sarmento TC, Costa EM, Martins CC, Granville-Garcia AF, Paiva SM. Impact of oral health conditions on the quality of life of preschool children and their families: a cross-sectional study. Health Qual Life Outcomes 2014;18;12:55. 
23. Dantas LR, Gomes MC, Dantas LR, Cruz-da-Silva BR, Perazzo MF, Siqueira MBLD, et al. The impact of dental treatment on oral health-related quality of life among preschool children. J Public Health 2015; 23(6):327-31.

24. Fiovarante DP, Marinho-Casanova ML. Comportamentos de crianças e dentistas em atendimentos odontológicos e profiláticos e de emergência. Interação em psicologia 2009; 13(1):147-54.

25. Ferreira JMS, Aragão AKR, Colares V. Técnicas de controle do comportamento infantil: revisão de literatura. Pesq Bras Odontoped Clin Integr 2009; 9(2):247-51.

26. Possobon RF, Carrascoza KC, Moraes ABA, Costa Jr. AD. O tratamento odontológico como gerador de ansiedade. Psicologia em Estudo 2007; 12(3):609-16.

27. Machado MS, Nagano HCM, Silva JYB, Bosco VL. Participação dos pais na tomada de decisões no atendimento odontológico de seus filhos. Revista de Odontologia da Universidade Cidade de São Paulo 2009;21(1):38-47.

28. de Castro AM, de Oliveira FS, de Paiva Novaes MS, Araújo Ferreira DC. Behavior guidance techniques in Pediatric Dentistry: attitudes of parents of children with disabilities and without disabilities. Spec Care Dentist 2013;33(5):213-7.

\section{Endereço para correspondência:}

Jainara Maria Soares Ferreira

Avenida Júlia Freire, 1200, sala 202, Expedicionários

58040040 João Pessoa-PB

Fone:83 35667794

E-mail: jainara.sf@gmail.com

Recebido: 22/06/2016. Aceito: 10/10/2016. 